\title{
Editorial
}

\section{Towards the Next Phase of the Journal of Numerical Cognition}

\author{
Wim Fias*a
}

[a] Ghent University, Ghent, Belgium.

Journal of Numerical Cognition, 2019, Vol. 5(3), 260-261, https://doi.org/10.5964/jnc.v5i3.272

Published (VoR): 2019-12-20.

*Corresponding author at: Department of Experimental Psychology, Faculty of Psychology and Educational Sciences, Henri Dunantlaan 2, 9000 Ghent, Belgium. E-mail: wfias@jnc.psychopen.eu

(c) (i) (his is an open access article distributed under the terms of the Creative Commons Attribution 4.0 International License, CC BY 4.0 provided the original work is properly cited.

Typically, a newly appointed editor writes an editorial to specify his or her goals and ambitions, additionally formulating a few words of thanks to the previous editor. I will do the opposite. I will extensively thank John Towse, the previous and founding editor-in-chief of the Journal of Numerical Cognition. As an aside, then, I will briefly describe the future of the journal.

John is the father of the journal. More than five years ago, John realised that the field of numerical and mathematical cognition had matured to such an extent that it might benefit from a place where specialised research could be presented to a specialised audience. John didn't only passively realise this. He also took action. Having explored possibilities, discussed pros and cons with colleagues, he founded the journal in 2015 through the PsychOpen GOLD platform (www.psychopen.eu), a publishing service and infrastructure provided by Leibniz Institute for Psychology Information (ZPID), Trier, Germany.

This wasn't without risk. An earlier attempt to create a journal dedicated to the field, Mathematical Cognition, launched in 1995, had turned out to be hard to sustain after a successful start. Apparently back in the 90's, the time wasn't ripe yet. John had a good intuition when sensing that this time was the right time. But, evidently, intuition is not enough. What has been the crucial pillar for the success is that he has invested a lot of time, dedication and energy in getting the journal off the ground.

Content-wise, John had felt that it was necessary to cover the full width of the domain of numerical cognition. From the beginning onwards, John has adhered to a broad scope for the journal. A broad scope in topics (from basic number representations to education), a broad scope in disciplines (from cognitive psychology to intervention research) and a broad scope in format (not only research reports but also, book reviews, commentaries and theoretical contributions). But there is one thing where John stuck to a narrow scope and that is quality. John has always guarded quality, which for a journal is extremely important: The reader must be sure that the paper she decides to read is of uncompromised quality. It is not easy to accomplish this. John did this with the 
composition of an excellent editorial board and with committed reviewers, and also, and most remarkably, through an honest and motivational attitude, encouraging authors, reviewers and editorial board members to invest efforts to increase quality.

John started his editorial in the first issue of the journal by taking the position of the research community asking 'Do we need yet another journal?' (Towse, 2015). Now, almost 5 years and 13 issues later, we can firmly answer "yes" to that question. At the time, the field had grown to such an extent that the general journals were not enough to give a place to the publication of specific developments in the field. A dedicated medium for publishing numerical cognition research was at stake. John felt it, took the right decisions and made it. We - and now I am speaking in the name of the community of numerical cognition researchers - cannot underestimate the importance of what John did for us. We owe him an awful lot of gratitude.

Around the same time that John started the journal, the need for coordination and a dedicated platform for interaction within the community of numerical and mathematical cognition researchers had also led to the foundation of a dedicated society, the Mathematical Cognition and Learning Society (MCLS). Soon, John and the MCLS, in interaction with PsychOpen, decided to join forces and in 2017 it was decided that the Journal of Numerical Cognition would become the official journal of the society.

Understandably after all the efforts he had done to bring the journal to where it is now, John decided that it was time to resign as the editor-in-chief of the journal. Once he had announced that, the MCLS has assigned me (being a member of the publications committee of the MCLS), the role of interim editor-in-chief with the general task of further elaborating and specifying the interactions between the MCLS, the journal and the publisher. More specifically, my main goals will be: to explicitly define the plans and ambitions of the society with respect to our journal; to bring these in correspondence with services and support that are provided by the publisher; to incorporate the goals of PsychOpen for open science (open access to sources and data) in the ambitions of the MCLS and to help define the procedure for selecting the editor-in-chief. Hence, my main goals for now are of a supportive nature, in such a way that the next editor can take over in a context in which the more formal aspects are settled so she or he can concentrate on the content-based aspects of the journal. For now, there is no need to concentrate on content-based aspects given the current good shape of the journal.

Compared to what John did for the journal, my contribution will be small. It is my honour and pleasure to continue the groundbreaking work of John Towse by laying out the foundations for the future maturation and development of the journal.

\section{References}

Towse, J. (2015). Finding a voice for numerical cognition. Journal of Numerical Cognition, 1(1), 1-6.

https://doi.org/10.5964/jnc.v1i1.16 